Rev. SINAPSIS, Edición № 10, Vol. 1, Junio 2017

\title{
La apatía a las matemáticas en las carreras universitarias humanísticas
}

\author{
Julio César Silva Ruiz MSc.; Alex Hugo Ramos Mendoza MSc.; Francisco Isaac \\ Verduga Vélez MSc.
}

Carrera de Finanzas. Universidad San Gregorio de Portoviejo. Ecuador

Contacto: jcsilva@sangregorio.edu.ec

Receptado: 24/03/2017

\section{Resumen}

Es frecuente escuchar en reuniones informales sobre la asignatura de matemáticas y el pasado estudiantil, en algunos casos se suele tener respuestas que reflejan apatía a la asignatura, expresada en experiencias poco agradables, llegando hasta seleccionar carreras que no tengan que ver con números ni cálculos matemáticos. Este ha sido motivo de estudio para los autores de la investigación, quienes indagaron en estudiantes universitarios para conocer en detalle, si la apatía está relacionada con estudiar carreras que tengan en el currículo la menor cantidad de asignaturas que involucren las matemáticas. El objetivo de este trabajo es determinar las causas de la apatía a las matemáticas en las carreras universitarias humanísticas, para lo cual se utilizó como instrumento de investigación la encuesta a estudiantes de las universidades: Técnica de Manabí y San Gregorio de Portoviejo, en las carreras de Trabajo Social y Jurisprudencia, identificando la principal debilidad de los docentes de matemáticas el homogenizar su metodología de trabajo, sin considerar que los grupos de estudios, y los estudiantes son distintos, añadiendo también que realizan diagnósticos inadecuados, que los textos a pesar de traer lecturas motivadoras la actitud de los docentes no contribuyen a una verdadera motivación, generando indiferencia y rechazo a la asignatura, concluyendo que la apatía y poco entusiasmo por la asignatura de Matemática es responsabilidad metodológica de los docentes, los cuales muestran insuficiencias pedagógicas para impartir la cátedra objeto de estudio de esta investigación.

Palabras claves: Didáctica de las Matemáticas, métodos de enseñanza de Matemáticas, Motivación hacia las Matemáticas, Hábitos de estudios.

\section{Summary}

Is often heard in informal meetings about the subject of mathematics and the past student, on some cases it is usually have answers that reflect apathy to the subject, express on unpleasant experiences, until you select careers that do not have to do with numbers or mathematical calculations. This has been reason of study for the authors of the investigation, who inquired about university students to know in detail, if the apathy is related with study careers that they have in the curriculum the least amount of subjects that involve the mathematics. The object of this work is determining the causes of the apathy to the mathematics in the university humanistic careers, for which the survey of university students was used as a research tool: Technical of Manabí and San Gregorio of Portoviejo, in the careers of Social Work and jurisprudence, identifying the 
main weakness of mathematics teachers to homogenize their work methodology, without considering that the group of studies, and the students are different, also adding that they make inadequate diagnoses, that the texts in spite of bringing motivational readings the attitude of the teachers do not contribute to a true motivation, generating indifference and rejection to the subject, concluding that apathy and little enthusiasm for the subject of mathematics is methodological responsibility of the teachers, which show pedagogical inadequacies to teach the lecture subject of study of this research.

Keywords: Didactics of the Mathematics, teaching methods of Mathematics, Motivation towards Mathematics, Study Habits.

\section{Introducción}

En los actuales momentos, los estudiantes al realizar el escogitamiento de su futura carrera universitaria optan por alejarse de aquellas que les dedican más tiempo a las ciencias exactas, evidenciando cierta apatía por esta ciencia, siendo este el problema científico que motivó la investigación, ante lo cual se buscó las causas por las cuales se produce el alejamiento del mundo de las matemáticas, cuando llega el momento de elegir una carrera universitaria.

La investigación radicó en identificar el origen de esa apatía que tienen los estudiantes por los números, concretamente. El término apatía significa desgano, desmotivación, falta de emociones o incluso enfermedad (Real Académia Española, 2001); desde que el escolar tiene los primeros contactos e intenta realizar ejercicios con operaciones sencillas y se siente tenso ante aquellas explicaciones que en los centros de estudios no le encuentran sentido a lo que explican los profesores de esta área, el refuerzo o esfuerzo de los padres de familia en casa, se ve frustrada muchas veces por la poca ayuda que ellos les puedan bridar, ya que también tuvieron inconvenientes numéricos.

Las universidades en su oferta académica, ofrecen carreras del área social o humanística, alejadas supuestamente del área matemática, motivando a aquellos estudiantes que buscan carreras en las que "no se estudien los números", sin percatarse que en el sistema admisión de las universidades ecuatorianas, en los exámenes de ingreso hay un componente lógico matemático que es el que marca el mayor puntaje para poder acceder a cualquier carrera universitaria, ante lo cual el currículo y la metodología para su desarrollo en el nivel del bachillerato requiere un replanteamiento general que motive al estudiantes no solamente hacia las matemáticas sino una valoración completa de las ciencias básicas, destacando su utilidad e importancia en el ejercicio de cualquier profesión, lo que implica motivar al desarrollo de actitudes, valores y competencias que hagan del estudiante un ser humano cumplidor en el mercado laboral y fiel con sus responsabilidades ciudadanas. (Sales, 2010).

Es importante indicar que la matemática es una ciencia que ayuda a otras asignaturas a desarrollar el pensamiento lógico, siendo la estructura donde se levantan otras ciencias; en los tiempos de Platón el pensamiento científico estaba aliado a la construcción de un modelo que pueda sacar adelante a su país, por lo que se le consideraba el manjar de los sabios, basado en un estado ideal con ayuda de la dialéctica y las matemáticas; lo que traía consigo una serie de axiomas e hipótesis con validez y contradicciones de las 
mismas, dando lugar a un empoderamiento de las ciencias exactas por grandes sabios, la opción de unir asignaturas y formar un reto interdisciplinario ya tenía auge desde aquellos tiempos, en la que los filósofos se ayudaban por figuras geométricas como principales premisas para formar argumentos de las ideas que fluían de sus axiomas. Platón reconoció que las matemáticas eran el soporte del pensamiento, pero que jerárquicamente estaba ubicado debajo del conocimiento de las ideas, pero sobre la opinión (Torres \& Patricio, 1998).

En otros estudios realizados, el énfasis de la apatía por los números recae en las lecturas matemáticas, el desconocimiento al ejercitar un problema o alguna operación hacen acentuar más esta debilidad estudiantil, la escritura matemática pierde interés cuando no va de la mano con la lectura matemática adecuada, este tipo de metodologías radica en la poca utilización de este recurso con el grupo de estudiantes en los años académicos respectivos y no saltando estas ejecuciones, ya que con el pasar de los periodos académicos se presentarán otros docentes con profundas contradicciones que harán perder el apetito del estudiante por el mundo matemático. (Gardner, 1997)

Los avances tecnológicos, metodologías, calculadoras, computadoras, juegos lúdicos, software, pizarras didácticas entre otros, han conseguido ser las herramientas útiles para dar soluciones parciales o totales a problemas o ejercicios matemáticos de diferentes grados de dificultad, pero no son lo suficientemente necesarios como para poder utilizarlos en el aula de clase, en el momento de una evaluación, taller o laboratorio, ya que el requisito es conocer cómo se resuelve y aplicar de acuerdo a reglas, que solo el ser humano puede hacerlo, el avance de la tecnología informática, especialmente en el aspecto educativo, coinciden en pronosticar cambios radicales en las instituciones educativas (Peña \& Esteve-Mon, 2017).

Es de reconocer que el proceso para no perder el gusto por las matemáticas también descansa en los hábitos de estudios que han venido arrastrando los estudiantes, es lógico pensar que si no existe esta riqueza adquirida será un escollo muy duro de esquivar, e incluso hará pensar a los estudiantes que el matemático nace con ese don, o que es hereditario; es digno de recalcar que deben existir horarios que permitan un breve descanso para continuar con la motivación del docente de que se resolverán todos los ejercicios o problemas propuestos, el tener sus propios apuntes es otra fortaleza, ya que no es más que el interés del estudiante durante las clases de matemáticas y es la conceptualización adquirida por su propio esfuerzo.

Este trabajo agrupó todas estas premisas y que permitieron plantear una serie de preguntas, como: ¿Por qué los estudiantes pierden o han perdido su interés por las matemáticas?, ¿es el docente la persona adecuada para esta asignatura?, ¿son adecuados los modelos educativos y metodologías aplicadas?, ¿las herramientas utilizadas son aprovechadas por los estudiantes?, interrogantes que en el desarrollo de la investigación irán dando luces a probables soluciones.

Los programas de estudios en matemáticas deben ir enlazados con una acertada dirección del jefe de área matemática, no puede ser posible que existan grandes saltos de un año académico a otro, y que los maestros se inventen direccionamientos que no 
están en concordancia con el momento ni con el grupo de estudiantes a quienes impartirá la cátedra; grandes brechas entre estudiantes de la Costa y de la Sierra ecuatoriana que conlleva a exámenes del Ministerio de Educación con grandes sesgos; creciendo la apatía por los números en grupos minoritarios y mayoritarios en las regiones del país. El documento "Resultados de las pruebas censales SER Ecuador 2008" (Son pruebas que miden el rendimiento del bachiller ecuatoriano para ingresar a las universidades públicas), demuestra que el nivel de los aprendizajes de los estudiantes en matemática es muy bajo, así por ejemplo en el tercer año de bachillerato existen estudiantes regulares e insuficientes en un porcentaje de $81,96 \%$; le sigue el décimo año con $80,43 \%$ y el cuarto año con $68,43 \%$. El mayor porcentaje de estudiantes con notas excelentes se encuentra en séptimo año con 3,23\%. (Ecuador, 2009)

La falta de metodologías para transmitir a los estudiantes clases atractivas, motivadoras que impulsen a mejorar el rendimiento en la asignatura de matemáticas, es otro trabajo que nos demuestra los obstáculos que se presentan y que hacen incrementar el número de estudiantes con apatía por los números; al no existir cabida para la expresión de conceptualizaciones e ideas, al imponerse tácticas rígidas ante que estrategias dinámicas que nacen en el estudiante, lo que traería mejores resultados, pasando el docente a ser un guía de dicha ejecución y no el que impone su forma de hacer. Es necesario que los docentes entendamos que debemos evitar dar clases magistrales, existe un autoengaño en el docente ya que están convencidos de que todo lo dijo bien, y que quedaron claros los temas tratados (Litwin, 2011).

El tener en las instituciones educativas textos modelos para diferentes periodos académicos, hace que el docente se mecanice con la ejecución de los ejercicios y problemas, cumpliendo con la secuencia sin detenerse a cubrir los desfases que existen con el grupo de estudiantes que no es el mismo del periodo académico anterior; estos casos se complementan cuando las tareas extraescolares son para cumplir con el llenado de un libro, con un revisado superficial de cumplimiento, pero no de conocimiento. Este proceso hace creer a los estudiantes que van por buen camino, y en el momento de las evaluaciones los resultados son otros, lo que trae como consecuencias deserciones, problemas sociales y desidia a las matemáticas.

Esta investigación permite reflexionar, conocer lo que está pasando con el proceso de dotar a los estudiantes con las primeras informaciones matemáticas y transformarlas en números con símbolos y signos, o con letras haciendo de este primer momento, un feliz encuentro del pensamiento con los resultados finales, y que surjan nuevos interrogantes para nuevas aplicaciones matemáticas, y para todo ello se necesita cambiar; es preciso que la creatividad reine en el proceso matemático, que la estrategia domine a la táctica dura y rígida que nuestros docentes nos ofrecen, no todo debe ser la táctica, esta no conjuga con el cerebro, hace falta la sorpresa, la predicción, la creatividad, la estrategia acompañada de técnicas que permitan al estudiante estar atento a la información (Willis, 2009). 
La idea a defender en este trabajo fue que: "Las deficiencias metodológicas dificultan la inserción de estudiantes hacia las carreras técnicas y viabilizan en el acceso de los estudiantes en las carreras universitarias humanísticas evadiendo la asignatura de matemática". El objetivo fue determinar las causas de la apatía a las matemáticas en los estudiantes de las carreras universitarias humanísticas, las tareas científicas fueron:1) identificar las metodologías utilizadas por los docentes de matemáticas, 2) determinar el grado de motivación de los estudiantes con los textos utilizados, 3) analizar experiencias de aprendizajes de los estudiantes en matemáticas, 4) investigar la complejidad de los contenidos de la asignatura de matemáticas.

\section{Materiales y métodos}

Este es un estudio cualitativo de cohorte transversal, en el cual se utilizaron los métodos: descriptivo, que permitió caracterizar el objeto de estudio, el método analítico en el tratamiento de la información estadística y los resultados obtenidos, constituyéndose en el nodo principal para realizar las conclusiones, éstas últimas se construyeron bajo las directrices del método sintético. Se aplicaron encuestas a los estudiantes de dos carreras del área social de las universidades: Técnica de Manabí y San Gregorio de Portoviejo, ambas con sede principal en la ciudad de Portoviejo, en Ecuador. Las carreras profesionales en las cuales se obtuvo la muestra fueron: Trabajo Social y Jurisprudencia respectivamente. La población la constituyeron 192 estudiantes de la carrera de Trabajo Social y 655 de la carrera de Jurisprudencia, para el cálculo muestral se trabajó con un nivel de significancia del $95 \%$.

\section{Resultados}

En la recolección de datos se aplicaron encuestas, el formulario constó de ocho preguntas, la consulta y levantamiento de información de la muestra en las dos universidades fue realizada por los investigadores, de los cuales se hizo énfasis a cuatro ítems, que contribuyeron de manera esencial por el mayor grado de aporte a la investigación.

Figura No. 1 Criterios de rechazo a la asignatura de matemáticas

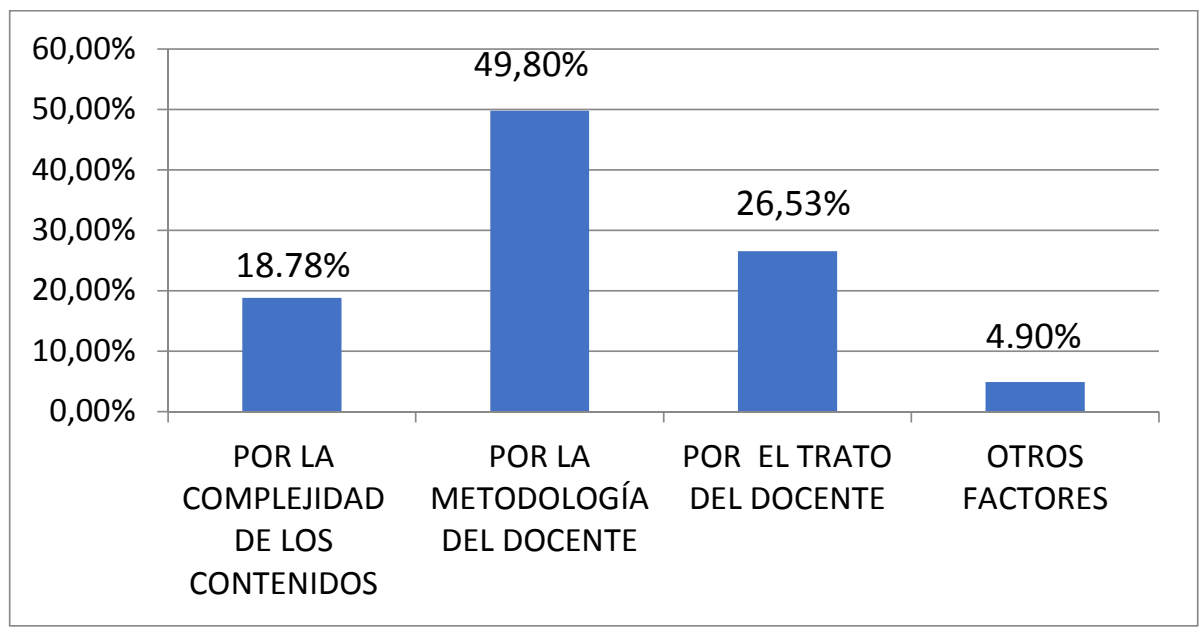


Rev. SINAPSIS, Edición № 10, Vol. 1, Junio 2017

Fuente: Estudiantes de la USGP y UTM

Después de tabular los resultados, estos aportaron significativamente a la investigación, es importante indicar que el docente para llegar al estudiante en la asignatura de matemáticas, requiere de un sinnúmero de herramientas metodológicas con características especiales ya que existen grupos con diagnósticos totalmente heterogéneos, es decir, que debe aplicarse la metodología adecuada con el grupo de estudiantes con el cual va a iniciar el proceso enseñanza - aprendizaje ; en la encuesta realizada los estudiantes involucrados nos indicaron que un profesor sin metodología hace que la asignatura no tenga importancia, el estudiante sienta antipatía hacia la misma y la excluya de sus preferencias, y que al ingresar a la universidad elijan carreras donde no tenga prioridad la parte numérica.

Figura No. 2 Criterios de mejoras para los docentes de matemáticas

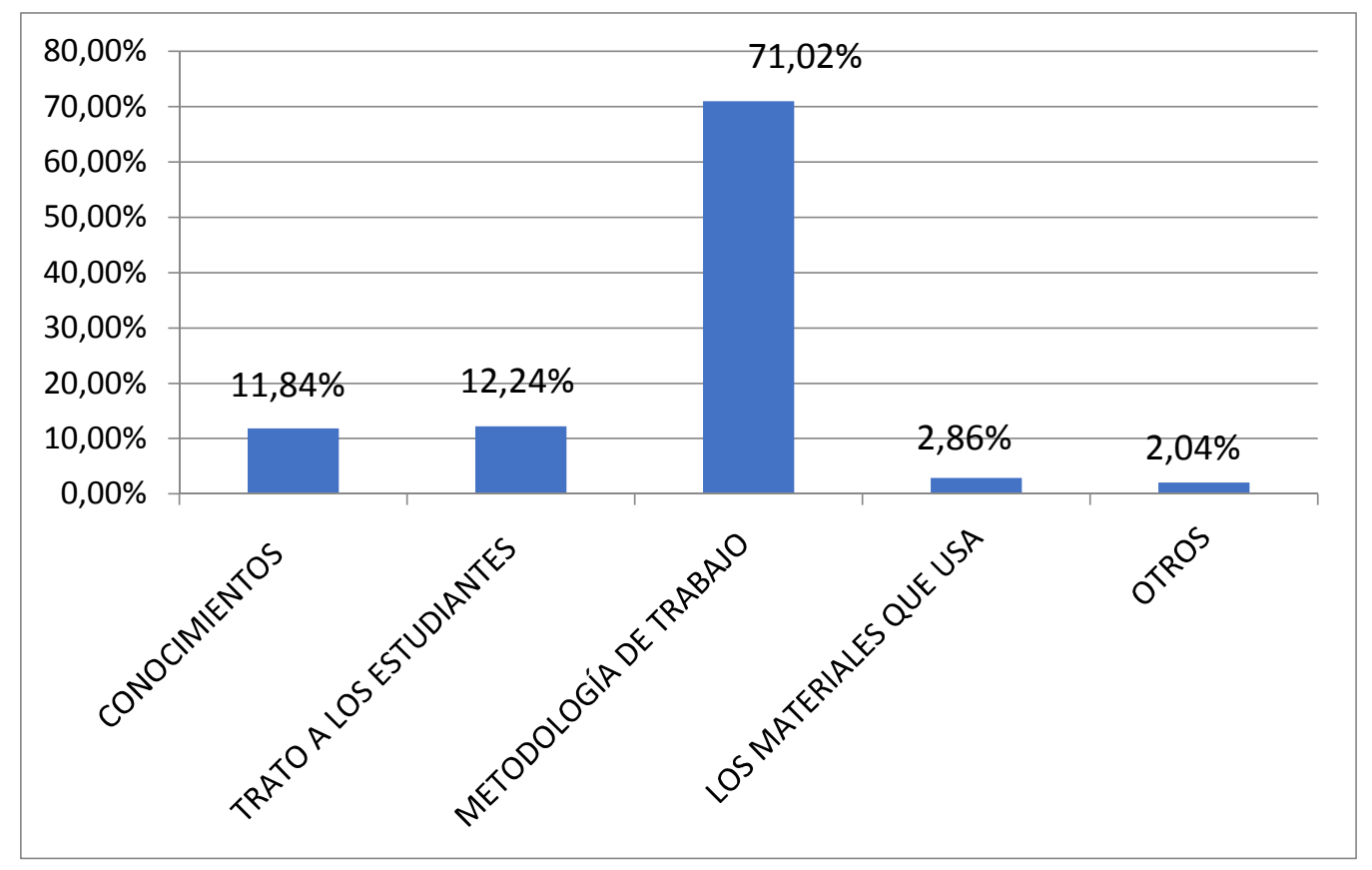

Fuente: Estudiantes de la USGP y UTM

Como consecuencia de la poca simpatía que presenta la asignatura de matemáticas en los estudiantes universitarios, estos nos revelaron en la encuesta que definitivamente lo que deben mejorar los docentes al momento de impartir la cátedra, es utilizar y aplicar una metodología capaz de involucrar al estudiante como principal protagonista, ya que muchas veces se puede carecer de herramientas o de un alto grado de conocimientos, pero si la metodología aplicada no guarda concordancia con el grupo, la atención de los estudiantes a la asignatura se pierde y por consiguiente crecerá la no aceptación de este componente educativo tan necesario. 
Figura No. 3 Los textos de matemáticas y su utilidad en la vida estudiantil

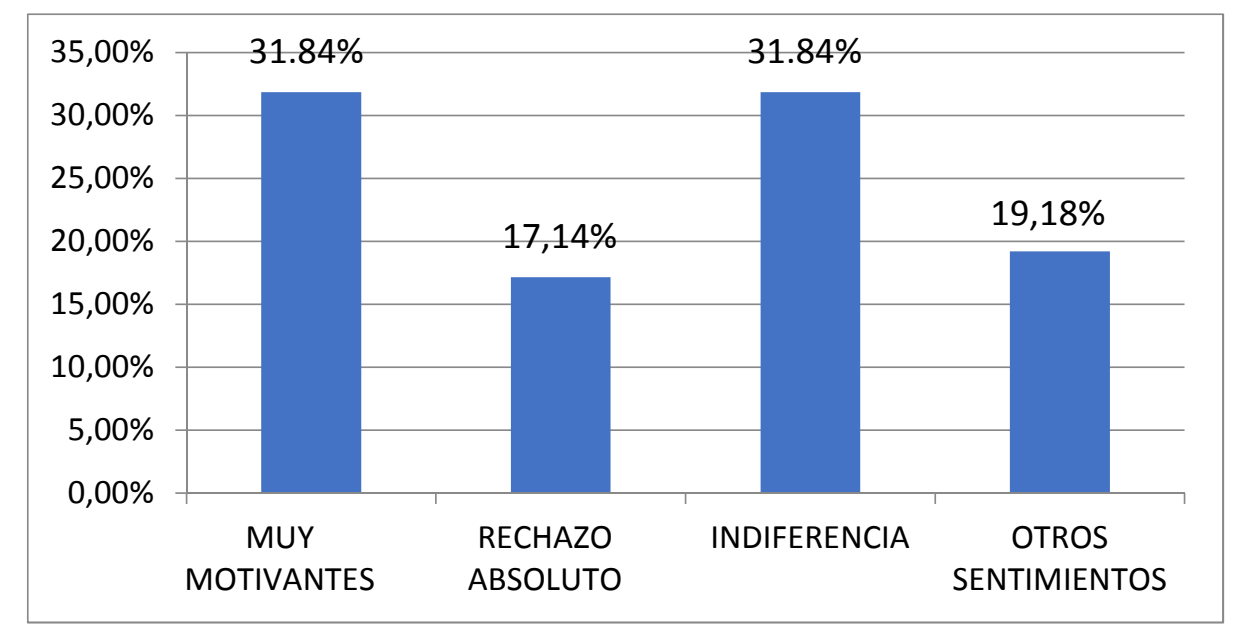

Fuente: Estudiantes de la USGP y UTM

Es necesario acotar, que el uso de los textos de matemáticas a pesar de ser muy motivantes para un grupo de los encuestados y con el mismo porcentaje a otro parte de la muestra le fue indiferente, es preocupante esta reacción de los estudiantes ya que consideraron que como todo texto intenta a sus lectores animarlos, para secuenciar dinámicamente la unidad o capítulo siguiente y su uso sea de aceptación en la mayoría de los lectores; esta reacción de los encuestados también se relación a las preguntas anteriores, no existe metodología , no existe razón para continuar, y el texto por muy motivante que sean no interesa al estudiante, y les sea indiferente e incluso de rechazo o cualquier otro sentimiento que no le permitan sentir simpatía hacia la asignatura.

Figura No. 4 Experiencia de aprendizaje de las matemáticas

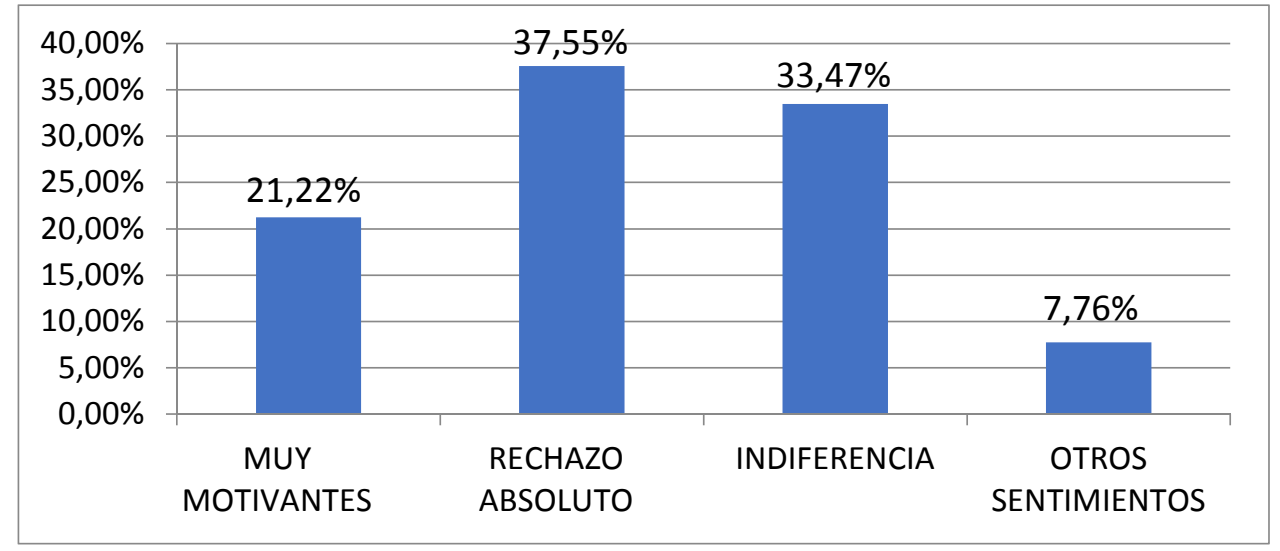

Fuente: Estudiantes de la USGP y UTM 
En esta experiencia de los estudiantes universitarios encuestados se refleja lo que ha sido como denominador común, rechazo absoluto e indiferencia, porcentajes que nos indican que existe apatía a las matemáticas, donde existe un gran número de estudiantes descontentos con su vida estudiantil, inconformes con un pasado que los llevó a elegir carreras universitarias donde no se aplique con regularidad la enseñanza de matemáticas.

\section{Discusión}

(Godino, Batanero, \& Vicenc, 2003), nos indican que surge una polémica muy interesante con la asignatura de matemáticas, que a pesar de ser tan importante para el desarrollo humano y científico, por qué es tan complicado para los estudiantes de cualquier ciclo educativo, obtener excelentes calificaciones. Y realiza el siguiente análisis, en primer lugar, el personal docente de esta área, no poseen las herramientas y métodos de enseñanza, la preparación que han tenido cada docente, puede ser que sean producto del mismo sistema educativo en que se educaron, lo cual imposibilita una transformación de metodologías, segundo, la actitud del docente al impartir la cátedra, en el transcurso de la misma, existen docentes con aires de superioridad, su ritmo es tan acelerado, que van dejando secuelas de dudas y profundos vacíos y con un trato hostil generando en el estudiante apatía no por los contenidos de la asignatura, sino por la persona encargada de la enseñanza, finalmente los aprendizajes matemáticos son acumulativos, de igual forma son las dificultades, es decir, que esos malestares de la primaria, siguen en secundaria y se vuelven insuperables a partir de la enseñanza superior (Hidalgo Alonso, Maaroto Saénz, \& Palacios Pico, 2005), lo que al comparar con la investigación realizada, los estudiantes reclaman nuevas metodologías acorde al momento en que están viviendo, desechar diagnósticos no reales, es fácil creer que existen contenidos que el estudiante ya conoce y por lo tanto seguir el formato trazado hace años atrás como lo más conveniente, y resulta que es todo lo contrario, es necesario que los docentes tengan otras herramientas para llegar al estudiante y sobre todo a una asignatura que necesita de metodologías innovadoras.

(Miranda, 2017), es otro aporte que nos narra bajo el tema: "Nos han estado enseñando mal las matemáticas durante todo este tiempo", el gran desatino que ha existido durante tanto tiempo, con tareas, ejercicios, problemas de largo metraje que fueron quienes condicionaron nuestras capacidades de desarrollar habilidades matemáticas, indica además que el verdadero proceso es de creatividad más que de velocidad; hace énfasis al talento del niño, que cultivando su creatividad y razonamiento serían las nuevas metodologías para hacer de las matemáticas el arte del ser humano, en la investigación indicaron los encuestados que no existe motivación lectora y el desánimo reina en las clases de matemáticas que a pesar de tener textos interesantes, nunca fueron utilizados para continuar con la escritura matemática, la importancia de una lectura matemáticas es necesaria para entender los ejercicios y poder resolver problemas.

(Pimienta, 2014), hace referencia que en la escuela muchas veces "matamos" al niño, al adolescente, al joven, y lo expresa en su libro titulado "Estrategias de enseñanza aprendizaje: docencia universitaria basada en competencias", no existe un espacio en el cual el docente se detenga a conocer con que conocimientos viene el estudiante, no 
existe la exploración, y la construcción del pensamiento se origina con lo que cree el docente que sabe del grupo que está tratando, en otros casos se da el conocimiento tratado sin que el estudiante tenga la oportunidad de intervenir y la metodología es de un grupo totalmente ajeno, es necesario que el nuevo grupo sea diagnosticado, para luego juntos plantear tácticas pero sobretodo crear estrategias para solucionar verdaderos problemas de la vida real, en la investigación, los estudiantes encuestados indicaron que la apatía a las matemáticas radica en grandes saltos que se dan en el sistema educativo nacional, falta de secuencias en los diferentes niveles por la que el estudiante hace la vida estudiantil, con la imperiosa necesidad de cambiar cada cierto periodos con sistemas educativos fuera de nuestra realidad y que no obedecen a la realidad.

Sobre el uso de textos para la asignatura de matemáticas, (Ochoviet, 2009) recomienda que los mismos tengan lecturas motivadoras para los estudiantes desde temprana edad, ya que también puede ser que en un texto de cualquier asignatura exista una lectura de tipo matemático, y que induce a que una metáfora matemática contribuya a la lectura de textos literatos. El aprender por medio de lecturas interesantes como cuentos, obras literarias, hace que el estudiante sienta respeto por el momento que está atravesando y lo motive a leer en forma comprensiva y escribir de igual manera, los harán sentir que forman parte de la historia, además de fortalecer la memoria, en la investigación realizada se corrobora con este aporte, ya que indiscutiblemente la asignatura de matemáticas no es solo ejercicios y problemas matemáticos, la simbología, formulas, momentos históricos son lecturas motivadoras, en las cuales las puntuaciones de lectura y escritura son necesarias para poder asimilar y crear un clima de predisposición ante la temática que se tratará en una clase de matemáticas.

\section{Conclusiones}

La apatía a las matemáticas en los estudiantes en las carreras universitarias humanísticas ha sido la principal causa, por la cual estos estudiantes no eligieron otras carreras, lo que fue comprobado a lo largo de la investigación realizada. Dando posibles soluciones para que el docente de matemáticas pueda mejorar, con metodologías atractivas, motivadoras, capaz de involucrar al estudiante en el proceso de enseñanza aprendizaje.

Estos cambios en los docentes, deben de estar ligados desde que el estudiante realiza sus primeros números en sus estudios, es decir, cuidar con lecturas que relacionen la vida real con el proceso de aprendizaje que están pasando, sin olvidar, realizar siempre el respectivo diagnóstico para aplicar verdaderos temas que harán profundos cambios y transformarse en la motivación que tiene el estudiante al llegar al aula escolar.

Todas las contribuciones de los diversos autores nos llevan a reflexionar, sobre el verdadero significado de estudiar alguna carrera que tenga como componente educativo las matemáticas, y por qué en el mundo existen pocos talentos para enseñar esta asignatura, y si existen, en las aulas son contados los que quieren modificar las metodologías al dar una cátedra de matemáticas; estas vivencias y aportes hacen que los gobiernos de turno encargados de encausar la educación en nuestro país ponga al frente del Ministerio de Educación en nuestro caso, a personas conocedoras de la realidad y que lleven soluciones para mejorar esta problemática. 
Rev. SINAPSIS, Edición № 10, Vol. 1, Junio 2017

\section{Bibliografía}

(2001). Real Académia Española. En Diccionario de la lengua española. Madrid.

Ecuador, M. d. (2009). Cómo interpretar los resultados. Resultados de las pruebas censales SER ECUADOR 2008, 8-11.

Gardner, H. (1997). Arte, mente y cerebro. Argentina: Paidós.

Godino, J., Batanero, C., \& Vicenc, F. (2003). Fundamentos de la enseñanzay el aprendizaje de las matemáticas. Matemáticas y su didáctica para maestros, 7121.

Hidalgo Alonso, S., Maaroto Saénz, A., \& Palacios Pico, A. (2005). El perfil emocional matemático como predictor de rechazo escolar:relación con las destrezas y los conocimientos desde una perspectiva evolutiva. Educación Matemática, 90.

Litwin, E. (2011). El Oficio de enseñar: condiciones y contextos. Buenos Aires: Paidós.

Miranda, B. (28 de abril de 2017). BBC Mundo. Recuperado el 25 de mayo de 2017, de http://www.bbc.com/mundo/noticias-39725468

Ochoviet, C. (2009). The Mathematics Enthusiast. Estados Unidos: Article.

Peña, E., \& Esteve-Mon, F. (2017). Educación, salud y TIC en contextos multiculturales: Nuevos espacios de intervención. En R. Zapata, D. Rachida, V. Cala, \& A. González, Innovación a la enseñanza (págs. 52-63). Almeria: Edual.

Pimienta, J. (2014). Estrategias de enseñanza aprendizaje: Docencia Universitaria basada en competencias. Colombia: Pearson.

Sales, A. (2010). La formación intercultural del profesorado:hacia la transformación social. Revista Latinoaméricana de educación inclusiva, 65-70.

Torres, L., \& Patricio, R. (1998). Los suicidios de Platón. Santiago de Chile: LOM.

Willis, J. (2009). Estrategias para convertir la información en conocimiento. Iberoamérica Divulga, 44-49. 